\title{
IMAGE DENOISING IN MULTIPLICATIVE NOISE
}

\section{Chandra Sekhar Seelamantula*}

\author{
Department of Electrical Engineering \\ Indian Institute of Science \\ Bangalore - 560 012, Karnataka, India \\ chandra.sekhar@ieee.org
}

\section{Thierry Blu}

\author{
Department of Electronic Engineering \\ The Chinese University of Hong Kong \\ Sha Tin, New Territories, Hong Kong \\ thierry.blu@m4x.org
}

\begin{abstract}
We address the problem of denoising images corrupted by multiplicative noise. The noise is assumed to follow a Gamma distribution. Compared with additive noise distortion, the effect of multiplicative noise on the visual quality of images is quite severe. We consider the mean-square error (MSE) cost function and derive an expression for an unbiased estimate of the MSE. The resulting multiplicative noise unbiased risk estimator is referred to as MURE. The denoising operation is performed in the wavelet domain by considering the image-domain MURE. The parameters of the denoising function (typically, a shrinkage of wavelet coefficients) are optimized for by minimizing MURE. We show that MURE is accurate and close to the oracle MSE. This makes MUREbased image denoising reliable and on par with oracle-MSEbased estimates. Analogous to the other popular risk estimation approaches developed for additive, Poisson, and chisquared noise degradations, the proposed approach does not assume any prior on the underlying noise-free image. We report denoising results for various noise levels and show that the quality of denoising obtained is on par with the oracle result and better than that obtained using some state-of-the-art denoisers.
\end{abstract}

Index Terms - multiplicative noise, unbiased risk estimation, Gamma distribution, speckle noise.

\section{INTRODUCTION}

In imaging modalities such as ultrasound imaging, synthetic aperture RADAR, SONAR, laser Doppler imaging, the image is reconstructed by a coherent demodulation of the incident electromagnetic or acoustic waves. The noise in these imaging modalities is neither additive nor Gaussian. The complex wavefield incident on each resolution cell of the imaging device consists of a real part (in-phase component) and an imaginary part (the quadrature-phase component) corresponding to

\footnotetext{
${ }^{*}$ C. S. Seelamantula was supported by the DRDO-IISc Frontiers Research Program and Robert-Bosch Centre for Cyberphysical Systems, IISc.

${ }^{\dagger}$ T. Blu was supported by the General Research Fund CUHK410012 from the Hong Kong Research Grant Council.
}

each scatterer on the object. Assuming a large number of randomly distributed scatterers, the net complex wavefield can be modeled as comprising of Gaussian distributed real and imaginary parts with zero mean and identical variances. The net magnitude follows a Rayleigh distribution and the intensity (or magnitude-squared measurement) follows an exponential distribution. The measurement can be expressed as the product of a reflectance function (the clean underlying image) and an exponential random variable of unit mean, which is the noise. The image thus measured has a granular appearance, which is referred to as speckle. For an excellent article on the properties of speckle, we refer the reader to the seminal articles by Goodman [1,2]. The goal in denoising such images is to estimate the reflectance function from the noisy measurement. The signal-to-noise ratio (SNR) of an image corrupted by exponentially distributed multiplicative noise is unity (that is, $0 \mathrm{~dB}$ ). In order to increase the SNR, one often considers multiple acquisitions of the same object to average out the effect of noise. Effectively, the reflectance function remains invariant across acquisitions and the noise keeps changing. Consequently, the averaged multi-look measurement is effectively equivalent to the reflectance image multiplied by the average of many independent and exponentially distributed random variables, which turns out to be a Gamma distribution. In this generic case, the measured image is expressed as the product of an unknown reflectance image multiplied by a Gamma distributed random variable. The SNR in this case increases to $k$, which is the number of looks/measurements.

\subsection{Prior art}

Some early work on adaptive algorithms for the restoration of images corrupted with speckle was reported by Kuan et al. [3], Lee [4], Frost et al. [5], Lopes et al. [6]. Recently, many approaches have been developed within a variational framework. The image is often assumed to be piecewisesmooth and a Bayesian framework is used to impose the prior. Approaches based on Markov random field priors were reported by Bioucas-Dias [7] and Oliver and Quegan [8]. Total variation (TV) regularization approaches for multiplicative noise suppression were proposed by Rudin et al. [11], Aubert 
and Aujol [9], Huang et al. [10], Shi and Osher [12], [13]. The TV approach adapts locally to the underlying image structure and results in piecewise-smooth estimates of the reflectance function. The variational and maximum a posteriori formulations give rise to non-convex data fidelity costs, which pose difficulties from an optimization perspective. An analysis of various data fidelity terms for the multiplicative noise model was given by Steidl and Teuber [14]. They also showed that considering the logarithm of the reflectance is also the most suitable formalism under multiplicative noise and TV regularization. The logarithm of the reflectance function was also considered by many authors, for example $[10,15,16]$. Bioucas-Dias and Figueredo [16] proposed a variable splitting method to solve the denoising problem. They use the alternating direction method of multipliers (ADMM) to solve the resulting constrained optimization problem.

\subsection{This paper}

We deploy the mean-square error (MSE) cost and develop an unbiased risk estimator of the MSE, which we shall refer to as the multiplicative noise unbiased risk estimator (MURE). The risk estimation approach resulted in robust denoising performance in the case of additive Gaussian noise [17], Poisson noise encountered in microscopy and other low-light imaging applications [18], and chi-squared noise encountered in magnetic resonance imaging applications [19]. We develop MURE analogously but taking into account the aspects that are unique to the multiplicative noise scenario. We consider denoising using undecimated filterbank transforms and optimize for the subband regression coefficients by optimizing MURE. We show that the MURE-optimal performance is close to the oracle MSE performance.

\section{PROBLEM FORMULATION}

We consider the problem setting in the generic case of Gamma distributed multiplicative noise. The image is represented in the vectorized form with each element expressed as $y_{i}=x_{i} w_{i}$, for $i=1,2, \cdots, N$, where $y_{i}$ denotes the $i^{\text {th }}$ element of the vector $\mathbf{y}$. The vector $\mathbf{y}$ is the noisy version of the original unknown image $\mathrm{x} \in \mathcal{R}^{+N}$. The noise vector is given as $\mathbf{w}=\left[w_{1}, w_{2}, \cdots, w_{N}\right] \in \mathcal{R}^{+N}$, with $i^{\text {th }}$ element $w_{i}$ following the distribution $q\left(w_{i}\right)=\frac{a^{b}}{\Gamma(b)} w_{i}^{b-1} \exp ^{-w_{i} a}$, where $a$ and $\frac{1}{b}$ are the scale and shape parameters, respectively. $q\left(w_{i}\right)$ has mean $\mathcal{E}\left[q\left(w_{i}\right)\right]=a / b$, and variance $\sigma_{w}^{2}=\mathcal{E}\left\{\left[q\left(w_{i}\right)-\mathcal{E}\left(q\left(w_{i}\right)\right)\right]^{2}\right\}=\frac{a}{b^{2}}$. For the $k$-look acquisition scenario, we have $a=b=k$. Let $\mathbf{f}: \mathcal{R}^{+N} \rightarrow \mathcal{R}^{+N}$ be the denoising function that yields an estimate of the reflectance function: $\hat{\mathbf{x}}=\mathbf{f}(\mathbf{y})$. The goal is to optimize the denoising function such that the mean-square error $(\mathrm{MSE})=$
$\frac{1}{N} \mathcal{E}\left\{\|\hat{\mathbf{x}}-\mathbf{x}\|^{2}\right\}$ is minimized. The key contribution of this paper is to develop such an unbiased estimate, which would serve as a surrogate to the MSE.

\section{MULTIPLICATIVE NOISE UNBIASED RISK ESTIMATOR (MURE)}

Before proceeding with the development of the risk estimator, we introduce the following notation.

Notation. Given a 1-D function $f$, we define $\mathcal{M}$ by the following operator

$$
\mathcal{M} f(y)=k \int_{0}^{1} f(s y) s^{k-1} \mathrm{~d} s .
$$

For a multivariate function $\mathbf{f}(\mathbf{y})=\mathbf{f}\left(y_{1}, y_{2}, \ldots, y_{N}\right)$, we introduce the operator $\mathcal{M}_{i}$, which applies the operator $\mathcal{M}$ to the $i^{\text {th }}$ component of $\mathbf{f}(\mathbf{y})$ only. This notation is extended straightforwardly to multivariate vector functions $\mathbf{f}(\mathbf{y})=\left[f_{1}(\mathbf{y}), f_{2}(\mathbf{y}), \ldots, f_{N}(\mathbf{y})\right]^{\mathrm{T}}$ according to

$$
\mathcal{M} \mathbf{f}(\mathbf{y})=\left[\mathcal{M}_{1} f_{1}(\mathbf{y}), \mathcal{M}_{2} f_{2}(\mathbf{y}), \ldots, \mathcal{M}_{N} f_{N}(\mathbf{y})\right]^{\mathrm{T}} .
$$

Lemma 1. Consider a 1-D function $f$ such that $\mathcal{E}\{|f(y)|\}$ is finite. If $y=x w$ where $w$ is a multiplicative Gamma distributed random variable, with mean 1 and variance $1 / k$, then

$$
\mathcal{E}\{x f(y)\}=\mathcal{E}\{y \mathcal{M} f(y)\},
$$

where the expectations are taken over the realizations of $w$.

Proof. Letting $y=w x$, we have

$$
\begin{aligned}
\mathcal{E}\{y \mathcal{M} f(y)\} & =\int_{0}^{\infty} k w x \mathrm{~d} w \int_{0}^{1} f(s w x) q(w) s^{k-1} \mathrm{~d} s \\
& =k x \int_{0}^{1} s^{k-1} \int_{0}^{\infty} w f(s w x) q(w) \mathrm{d} w \mathrm{~d} s \\
& =k x \int_{0}^{1} s^{k-1} \int_{0}^{\infty} \frac{w}{s^{2}} f(w x) q\left(\frac{w}{s}\right) \mathrm{d} w \mathrm{~d} s \\
& =k x \int_{0}^{\infty} f(w x) \int_{0}^{1} \frac{w^{k}}{s^{2}} \frac{k^{k}}{\Gamma(k)} \mathrm{e}^{-k w / s} \mathrm{~d} s \mathrm{~d} w \\
& =k x \int_{0}^{\infty} f(w x) \frac{k^{k} w^{k}}{\Gamma(k)}\left[\frac{\mathrm{e}^{-k w / s}}{k w}\right]_{0^{+}}^{1} \mathrm{~d} w \\
& =x \int_{0}^{\infty} f(w x) \frac{k^{k} w^{k-1}}{\Gamma(k)} \mathrm{e}^{-k w} \mathrm{~d} w \\
& =x \int_{0}^{\infty} f(w x) q(w) \mathrm{d} w=\mathcal{E}\{x f(y)\} .
\end{aligned}
$$

The hypothesis $\mathcal{E}\{|f(y)|\}<\infty$ was used to validate the integration sign exchanges, and the limit at $0^{+}$.

Using Lemma 1, we derive an expression for the unbiased estimate of the risk as follows: 
Theorem 1. An unbiased estimate of the MSE (or risk) is given by the expression

$$
\operatorname{MURE}(\mathbf{f})=\frac{1}{N}\left(\frac{k}{k+1}\|\mathbf{y}\|^{2}+\|\mathbf{f}(\mathbf{y})\|^{2}-2 \mathbf{y}^{\mathrm{T}} \mathcal{M} \mathbf{f}(\mathbf{y})\right) .
$$

Proof. Since $\|\mathbf{f}(\mathbf{y})-\mathbf{x}\|^{2}=\|\mathbf{x}\|^{2}+\|\mathbf{f}(\mathbf{y})\|^{2}-2 \mathbf{x}^{\mathrm{T}} \mathbf{f}(\mathbf{y})$ we need to find two functions of $\mathbf{y}$ alone that are unbiased estimates of $\|\mathbf{x}\|^{2}$ and $\mathbf{x}^{\mathrm{T}} \mathbf{f}(\mathbf{y})$.

- First, $\mathbf{x}^{\mathrm{T}} \mathbf{f}(\mathbf{y})=x_{1} f_{1}(\mathbf{y})+x_{2} f_{2}(\mathbf{y})+\cdots x_{N} f_{N}(\mathbf{y})$ : From Lemma 1, we have that

$$
\mathcal{E}\left\{x_{i} f_{i}(\mathbf{y})\right\}=\mathcal{E}\left\{y_{i} \mathcal{M}_{i} f(\mathbf{y})\right\}
$$

which shows that

$$
\begin{aligned}
\mathcal{E}\left\{\mathbf{x}^{\mathrm{T}} \mathbf{f}(\mathbf{y})\right\}= & \mathcal{E}\left\{y_{1} \mathcal{M}_{1} f(\mathbf{y})\right\}+\mathcal{E}\left\{y_{2} \mathcal{M}_{2} f(\mathbf{y})\right\} \\
& +\cdots \mathcal{E}\left\{y_{N} \mathcal{M}_{N} f(\mathbf{y})\right\} \\
= & \mathcal{E}\left\{\mathbf{y}^{\mathrm{T}} \mathcal{M} \mathbf{f}(\mathbf{y})\right\}
\end{aligned}
$$

and so that $\mathbf{y}^{\mathrm{T}} \mathcal{M} \mathbf{f}(\mathbf{y})$ is an unbiased estimate of $\mathbf{x}^{\mathrm{T}} \mathbf{f}(\mathbf{y})$.

- Second, $\|\mathbf{x}\|^{2}=x_{1}^{2}+x_{2}^{2}+\cdots x_{N}^{2}$ : looking at the $i^{\text {th }}$ component of $\mathbf{y}, y_{i}=x_{i} w_{i}$, we have that $\mathcal{E}\left\{y_{i}^{2}\right\}=$ $x_{i}^{2} \mathcal{E}\left\{w_{i}^{2}\right\}=x_{i}^{2}(1+1 / k)$. Hence,

$$
x_{i}^{2}=\frac{k}{k+1} \mathcal{E}\left\{y_{i}^{2}\right\}, \text { and finally }\|\mathbf{x}\|^{2}=\frac{k}{k+1}\|\mathbf{y}\|^{2} .
$$

Therefore, the unbiased estimate of MSE is

$$
\operatorname{MURE}(\mathbf{f})=\frac{1}{N}\left(\frac{k}{k+1}\|\mathbf{y}\|^{2}+\|\mathbf{f}(\mathbf{y})\|^{2}-2 \mathbf{y}^{\mathrm{T}} \mathcal{M} \mathbf{f}(\mathbf{y})\right)
$$

\section{MURE-OPTIMIZED DENOISING USING UNDECIMATED FILTERBANK TRANSFORMS}

We perform transform-domain denoising of the image by considering the image-domain MURE. The denoising function $f$ is essentially specified as a combination of the analysis filters, transform-domain shrinkage, and the synthesis filters. Consider a $J$-band undecimated fiterbank transform with the analysis filters $\left\{\tilde{G}_{j}\left(z^{-1}\right), j=1,2, \cdots, J\right\}$ and synthesis filters $\left\{G_{j}\left(z^{-1}\right), j=1,2, \cdots, J\right\}$ (typically finite-impulse response filters). In matrix form, we express the analysis part as a huge matrix $\mathbf{D}=\left[\mathbf{D}_{1}^{T}, \mathbf{D}_{2}^{T}, \mathbf{D}_{3}^{T}, \cdots, \mathbf{D}_{J}^{T}\right](\mathbf{D}$ stands for multiband decomposition) and the reconstruction part as another matrix $\mathbf{R}=\left[\mathbf{R}_{1}, \mathbf{R}_{2}, \mathbf{R}_{3}, \cdots, \mathbf{R}_{J}\right]$ ( $\mathbf{R}$ stands for reconstruction). Each of the constituent matrices $\mathbf{D}_{j}$ or $\mathbf{R}_{j}$ is an $M \times M$ circulant matrix. The analysis and synthesis filterbanks form a perfect reconstruction pair, that is, $\mathbf{R D}=\mathbf{I}$, where $\mathbf{I}$ denotes the $J M \times J M$ identity matrix. Further, each submatrix is specified as $\mathbf{D}_{j}=\left[d_{m, \ell}^{j}\right]_{1 \leq m, \ell \leq M}, \mathbf{R}_{j}=$ $\left[r_{m, \ell}^{j}\right]_{1 \leq m, \ell \leq M}$, where $d_{m, \ell}^{j}=\sum_{n \in \mathbb{Z}} \tilde{g}_{j}[\ell-m+n M]$ and $r_{m, \ell}^{j}=\sum_{n \in \mathbb{Z}} g_{j}[\ell-m+n M]$, for $j=1,2, \cdots, J$. The analysis filterbank takes a vector input and generates a collection of vectors. The denoising operations are performed on the vectors and the synthesis block takes the collection of vectors and generates a vector. The overall denoising operation may be represented in a generic form as $\widehat{\mathbf{x}}=\mathbf{f}(\mathbf{y})=\mathbf{R} \boldsymbol{\theta}$ Dy. Corresponding to the input $\mathbf{y}$ (which is the vectorized noisy image), let the output of the analysis filterbank be $\left[\mathbf{w}^{1}, \mathbf{w}^{2}, \cdots, \mathbf{w}^{J},\right]$, Stating explicitly, $\mathbf{w}^{j}=\left[w_{m}^{j}\right]_{1 \leq m \leq M}$, where $w_{m}^{j}=\sum_{\ell=1}^{N} d_{m, \ell}^{j} y_{\ell}$. Considering the shrinkage parameters $\alpha^{j}$ for each subband, we write the $p^{\text {th }}$ pixel estimate in the $j^{\text {th }}$ subband as $\widehat{\mathbf{x}}_{p}^{j}=f_{\ell}^{j}(\mathbf{y})=$ $\sum_{m=1}^{M} r_{p, m}^{j} a_{j} w_{m}^{j}=\sum_{m=1}^{M} r_{p, m}^{j} a_{j} \sum_{\ell=1}^{M} d_{m, \ell}^{j} y_{\ell}$. In this paper, we shall consider only point-wise wavelet shrinkage mainly to illustrate the power of MURE. In a journal version, we shall consider a linear expansion of thresholds (LET) approach akin to that in [17-19]. The overall subband-adaptive transform-domain estimator is $\mathbf{f}(\mathbf{y})=\sum_{j=1}^{J} a_{j} \mathbf{f}^{j}(\mathbf{y})$, where $\mathbf{f}^{j}(\mathbf{y})=\left[f_{1}^{j}(\mathbf{y}) f_{2}^{j}(\mathbf{y}) \cdots f_{M}^{j}(\mathbf{y})\right]^{\mathrm{T}}$. The term $\|\mathbf{f}(\mathbf{y})\|^{2}$ in (7) can be expressed in the quadratic form $\mathbf{a}^{\mathrm{T}} \mathbf{M a}$, where $\mathbf{a}=\left[a_{1}, a_{2}, \cdots, a_{J}\right]^{\mathrm{T}}$ and $\mathbf{M}=\mathbf{f}_{\ell} \mathbf{f}_{\ell}^{\mathrm{T}}$, where, in turn, $\mathbf{f}_{\ell}=\left[f_{\ell}^{1}(\mathbf{y}), f_{\ell}^{2}(\mathbf{y}), \cdots, f_{\ell}^{J}(\mathbf{y})\right]^{\mathrm{T}}$. Next, we focus our attention on computing the term $\mathbf{y}^{\mathrm{T}} \mathcal{M} \mathbf{f}(\mathbf{y})$. Considering the $\mathcal{M} \mathbf{f}(\mathbf{y})$ term in (1), we compute

$$
\begin{array}{r}
\mathcal{M}_{\ell} f_{\ell}(\mathbf{y})=k \sum_{j=1}^{J} a_{j} \int_{0}^{1} f_{\ell}^{j}\left(y_{1}, y_{2}, \cdots, y_{\ell-1},\right. \\
\left.s y_{\ell}, y_{\ell+1}, \cdots, y_{N}\right) s^{k-1} \mathrm{~d} s,
\end{array}
$$

which upon simplification becomes

$$
\mathcal{M}_{\ell} f_{\ell}(\mathbf{y})=\sum_{j=1}^{J} a_{j} \sum_{m=1}^{M} r_{\ell, m}^{j} \alpha_{m}^{j}\left(w_{m}^{j}-\frac{d_{m, \ell}^{j}}{k+1} y_{\ell}\right) .
$$

The cross-term $\mathbf{y}^{\mathrm{T}} \mathcal{M} \mathbf{f}(\mathbf{y})$ in (7) is calculated as

$$
\begin{aligned}
\mathbf{y}^{\mathrm{T}} \mathcal{M} \mathbf{f}(\mathbf{y})= & \sum_{\ell=1}^{M} y_{\ell} \mathcal{M}_{\ell} \mathbf{f}_{\ell}(\mathbf{y}) \\
= & \sum_{j=1}^{J} a_{j} \sum_{\ell=1}^{M} y_{\ell} \sum_{m=1}^{M} r_{\ell, m}^{j} w_{m}^{j} \\
& -\frac{1}{k+1} \sum_{\ell=1}^{M} \underbrace{\left(\sum_{m=1}^{M} r_{\ell, m}^{j} d_{m, \ell}^{j}\right)}_{\text {constant }} y_{\ell}^{2},
\end{aligned}
$$




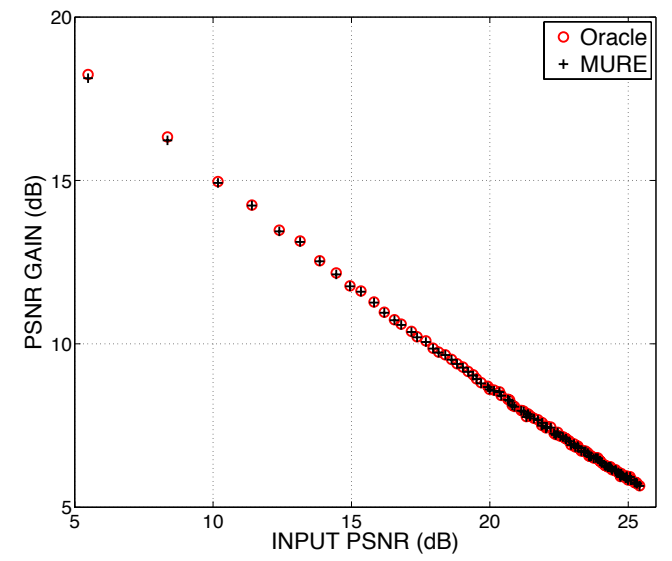

Fig. 1. Comparison of MURE versus oracle performance.

which can be expressed in the form $\mathbf{a}^{\mathrm{T}}\left(\sum_{\ell=1}^{M} y_{\ell} \mathbf{v}_{\ell}\right)$, where $\mathbf{v}_{\ell}=\left[f_{\ell}^{1}, f_{\ell}^{2}, \cdots, f_{\ell}^{J}\right]^{\mathrm{T}}$. The optimum coefficient vector $\mathbf{a}^{*}$ is obtained by setting the gradient of MURE with respect to $a$ to zero. This results in $\mathbf{a}^{*}=\mathbf{M}^{-1}\left(\sum_{\ell=1}^{M} y_{\ell} \mathbf{v}_{\ell}\right)$. The denoised image is obtained as $\mathbf{f}^{*}(\mathbf{y})=\left[\mathbf{a}^{\mathrm{T}} \mathbf{v}_{1}, \mathbf{a}^{\mathrm{T}} \mathbf{v}_{2}, \cdots, \mathbf{a}^{\mathrm{T}} \mathbf{v}_{M}\right]^{\mathrm{T}}$.

\subsection{Experimental results}

For the purpose of illustration, we consider the Champs image of size $256 \times 256$ from [16] and varied the look of the acquisition (equivalently, the SNR) progressively from 1 to 100. Denoising is performed using a four-level undecimated Haar wavelet transform. A comparison of PSNR gains obtained using MURE- and oracle-based approaches is shown in Figure 1. We observe that the PSNR gains are significantly higher at low input PSNR than at high input PSNR. Also, the gains provided by the MURE approach are very close to those obtained using the oracle method.

A comparison of the denoising performance of the MURE technique with two benchmark techniques ${ }^{1}$ in multiplicative image denoising literature, namely MIDAL [16] and the technique of Aubert-Aujol (AA) [9], is shown in Table 1. The MURE approach outperforms the AA technique by about 2 $\mathrm{dB}$, and MIDAL by about $1 \mathrm{~dB}$ in most cases. The denoised images corresponding to a 10-look acquisition are shown in Figure 2. The PSNR is the highest and the execution time is the least for the MURE approach. The oracle solution is also shown to serve as a reference. A thorough comparison for multiple images and using visual quality assessment metrics will be reported in a journal version of this manuscript.

\footnotetext{
${ }^{1}$ J. Bioucas-Dias, M. A. T. Figueiredo, J. F. Aujol, and G. Aubert kindly provided their MATLAB programs, which facilitated the comparisons.
}
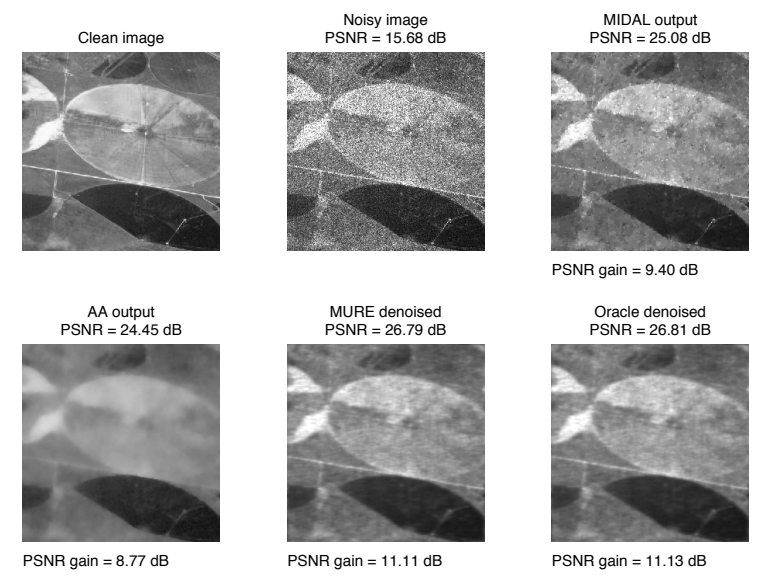

PSNR gain $=9.40 \mathrm{~dB}$

Oracle denoised
PSNR $=26.81 \mathrm{~dB}$

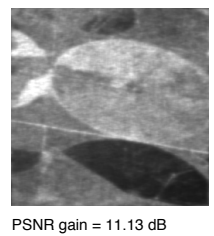

Fig. 2. Comparison of denoising performance of the proposed technique (image: Champs) with MIDAL and the technique of Aubert-Aujol. Matlab execution times: MIDAL: $3.2 \mathrm{~s}$; AA: $64.5 \mathrm{~s}$; MURE: $0.11 \mathrm{~s}$; Oracle: $0.11 \mathrm{~s}$.

Table 1. Comparison of PSNR improvement (in $\mathrm{dB}$ ) for the three techniques for various looks of acquisition $(k)$ on the standard Champs and Nimes images taken from [16].

\begin{tabular}{|c||c|c|c||c|c|c|}
\hline \multicolumn{1}{|c||}{} & \multicolumn{3}{c||}{ Champs } & \multicolumn{3}{c|}{ Nimes } \\
\hline$k$ & MIDAL & AA & MURE & MIDAL & AA & MURE \\
\hline 1 & 16.79 & 14.59 & 17.79 & 8.16 & 5.94 & 9.83 \\
3 & 14.47 & 13.28 & 14.57 & 5.69 & 5.22 & 6.88 \\
5 & 12.73 & 11.70 & 13.07 & 4.74 & 4.11 & 5.59 \\
7 & 11.17 & 10.34 & 12.20 & 4.28 & 3.09 & 4.83 \\
9 & 10.01 & 9.28 & 11.40 & 3.83 & 2.24 & 4.23 \\
\hline
\end{tabular}

\section{CONCLUSIONS}

We have addressed the problem of image denoising in multiplicative noise conditions and developed a new multiplicative noise unbiased risk estimation (MURE) approach for denoising. We developed a denoising solution in the undecimated Haar wavelet transform domain, whose performance turned out to be very close to that obtained using the oracle. A performance comparison with two benchmark techniques in the area showed superior denoising performance and least computational complexity. The denoising function chosen is linear and the corresponding MURE was evaluated in closedform directly in terms of the image pixels. With nonlinear denoising functions, the evaluation of the $\mathcal{M}$ operator in (1) would not be straightforward. The overall denoising performance is expected to be higher with nonlinear shrinkage functions. This aspect, together with a detailed experimental validation using visual quality assessment metrics will be reported separately. 


\section{REFERENCES}

[1] J. Goodman, "Some fundamental properties of speckle," Journal of the Optical Society of America, vol. 66, no. 1, pp. 1145-1150, 1976.

[2] J. Goodman, Speckle Phenomena in Optics: Theory and Applications. Greenwood Village, CO: Roberts, 2007.

[3] D. T. Kuan, A. A. Sawchuk, T. C. Strand, and P. Chavel, "Adaptive restoration of images with speckle," IEEE Transactions on Acoustics, Speech, and Signal Processing, vol. ASSP-35, pp. 373-383, Feb. 1987.

[4] J. S. Lee, "Digital image enhancement and noise filtering by use of local statistics," IEEE Transactions on Pattern Analysis and Machine Intelligence, vol. PAMI-2, pp. 165-168, Mar 1980.

[5] V. S. Frost, J. A. Stiles, K. S. Sanmugan, and J. C. Holtzman, "A model for radar images and its application to adaptive digital filtering of multiplicative noise," IEEE Transactions on Pattern Analysis and Machine Intelligence, vol. PAMI-4, pp. 157-165, 1982.

[6] A. Lopes, E. Nezry, R. Touzi, and H. Laur, "Structure detection and statistical adaptive speckle filtering in SAR images," International Journal of Remote Sensing, vol. 14, pp. 1735-1758, 1993.

[7] J. Bioucas-Dias, T. Silva, and J. Leitão, "Adaptive restoration of speckled SAR images," Proceedings of the IEEE International Geoscience and Remote Sensing Symposium (GRSS), vol. I, pp. 19-23, 1998.

[8] C. Oliver and S. Quegan, Understanding Synthetic Aperture Radar Images. Artech House, 1998.

[9] G. Aubert and J. Aujol, "A variational approach to remove multiplicative noise," SIAM Journal on Applied Mathematics, vol. 68, pp. 925-946, 2008.

[10] Y. Huang, M. Ng, and Y. Wen, "A new total variation method for multiplicative noise removal," SIAM Journal on Imaging Sciences, vol. 8, pp. 11-49, 1991.

[11] L. Rudin, P. Lions, and S. Osher, "Multiplicative denoising and deblurring: Theory and algorithms," Geometric Level Set Methods in Imaging, Vision, and Graphics, pp. 103-119, Springer, 2003.

[12] J. Shi and S. Osher, "A nonlinear inverse scale method for a convex multiplicative noise model," SIAM Journal on Imaging Sciences, vol. 1, pp. 294-321, 2008.

[13] D. -Q. Chen and L. -Z. Cheng, "Spatially adapted total variation model to remove multiplicative noise," IEEE Transactions on Image Processing, vol. 21, no. 4, Apr. 2012.
[14] G. Steidl and T. Teuber, "Removing multiplicative noise by Douglas-Rachford splitting methods," Journal of Mathematical Imaging and Vision, 2009.

[15] S. Durand, J. Fadili and M. Nikolova, "Multiplicative noise removal using $\ell_{1}$ fidelity on frame coefficients," Journal of Mathematical Imaging and Vision, vol. 36, no. 3, pp. 201-226, 2009.

[16] J. Bioucas-Dias and M. Figueiredo, "Multiplicative noise removal using variable splitting and constrained optimization," IEEE Transactions on Image Processing, vol. 19, no. 7, pp. 1720-1730, July 2010.

[17] T. Blu and F. Luisier, "The SURE-LET approach to image denoising," IEEE Transactions on Image Processing, vol. 16, no. 11, pp. 2778-2786, Nov. 2007.

[18] F. Luisier, C. Vonesch, T. Blu, and M. Unser, "Fast interscale wavelet denoising of Poisson-corrupted images," Signal Processing, vol. 90, no. 2, pp. 415-427, Feb. 2010 .

[19] F. Luisier, T. Blu, and P. J. Wolfe, "A CURE for noisy magnetic resonance images: Chi-square unbiased risk estimation," IEEE Transactions on Image Processing, vol. 21, no. 8, Aug. 2012. 